\title{
ON NECESSARY AND SUFFICIENT CONDITIONS FOR THE CONVERGENCE OF THE RENEWAL DENSITY
}

BY

\author{
WALTER L. SMITH( $\left.{ }^{1}\right)$
}

1. Introduction. If $k(x)$ is any non-negative function in $L_{1}(-\infty,+\infty)$, and if we write $k^{* 1}(x)=k(x)$ and

$$
k^{* n}(x)=\int_{-\infty}^{+\infty} k^{*(n-1)}(x-z) k(z) d z
$$

for $n=2,3, \cdots$, then the function

$$
\Delta[k(x)] \equiv \sum_{n=1}^{\infty} k^{* n}(x)
$$

is defined almost everywhere (although it is possibly infinite for some, or even almost all, $x$ ).

Suppose $f(x)$ is the probability density function of a random variable $X$, the mean value of which $\mu_{1}=\varepsilon X_{1}$, is strictly positive (it may have the value $+\infty)$. The function $\Delta[f(x)]$ is called the renewal density, and its asymptotic behavior has been an object of study since the earliest days of renewal theory. Interest was centered upon establishing (or denying) that as $x \rightarrow+\infty$,

$$
\Delta[f(x)] \rightarrow \mu_{1}^{-1} .
$$

Prior to the important paper of Feller [3] there appears to have been some controversy concerning this asymptotic behavior. Feller provided sufficient conditions under which the renewal density would converge to the desired limit. These conditions have been modified and simplified by Täcklind [10] and by Smith $[6 ; 7]$. The simplest sufficient conditions to date are those given by Smith [7]; these are: (i) $f(x) \rightarrow 0$ as $x \rightarrow+\infty$; (ii) $f(x) \in L_{1+\delta}$ for some $\delta>0$.

In the course of some work on the theory of dams we have recently discovered that if

$$
\begin{array}{rlrl}
f(x) & =\int_{0}^{\infty} \frac{e^{-(t+x)} x^{t-1}}{\Gamma(t)} d t, & x \geqq 0, \\
& =0, & & x<0,
\end{array}
$$

Received by the editors September 5, 1961.

(1) This research was supported by the Office of Naval Research under contract No. Nonr-855(09) for research in probability and statistics at the University of North Carolina, Chapel Hill, N. C. Reproduction in whole or in part is permitted for any purposes of the United States Government. 
then $\mu_{1}=1$ and $\Delta[f(x)] \rightarrow 1$, so that the renewal density does converge to the appropriate limit. However, $f(x)$ is in no class $L_{1+\delta}$ for $\delta>0$. Thus the sufficient conditions of Smith [7] are not necessary. We refer to Smith [8] for a discussion of this example.

This paper is mainly concerned with establishing that a certain set of conditions on $f(x)$ is both necessary and sufficient for the asymptotic convergence of the renewal density. Before we state this main theorem, however, we must introduce some notation.

Let $U(x)=P\{0 \leqq x\}$ be the Heaviside unit function; i.e., $U(x)=1$ if $x \geqq 0$ and $U(x)=0$ if $x<0$. For any $\delta>0$ define $c_{\delta}(x)=f(x) U(x-\delta)$, and $b_{\delta}(x)$ $=f(x) U(x)-c_{\delta}(x)$; then define $a(x)=f(x)-b_{\delta}(x)-c_{\delta}(x)$. Thus $a(x)$ vanishes for all positive $x, b_{\delta}(x)$ vanishes outside the interval $[0, \delta)$, and $c_{\delta}(x)$ vanishes whenever $x<\delta$; for all $x$, however, $f(x)=a(x)+b_{\delta}(x)+c_{\delta}(x)$. Denote Fourier transforms thus

$$
c_{\delta}^{\dagger}(\theta)=\int_{-\infty}^{+\infty} e^{i \theta x} c_{\delta}(x) d x=\int_{\delta}^{\infty} e^{i \theta x} f(x) d x
$$

We can now state,

THEOREM 1. If $f(x)$ is the probability density function of a random variable $X$ with a mean value $\mu_{1}=\varepsilon X$ which is strictly positive (though, possibly infinite), then in order that $\Delta[f(x)] \rightarrow \mu_{1}^{-1}$ as $x \rightarrow \infty$, where $\mu_{1}^{-1}$ is to be interpreted as zero if $\mu_{1}=+\infty$, it is necessary and sufficient that

(i) $f(x) \rightarrow 0$ as $x \rightarrow+\infty$, and, for some $\delta>0$,

(ii) $\Delta\left[b_{\delta}(x)\right] \rightarrow 0$ as $x \rightarrow \infty$,

(iii) $c_{\delta}^{\dagger}(\theta)$ belongs to some class $L_{p}$, where $p$ may depend upon $\delta$. Furthermore, if these conditions hold for some $\delta>0$, then they hold for every $\delta>0$ for which

$$
\int_{0}^{\delta} f(x) d x<1
$$

This is the first time that necessary and sufficient conditions for the convergence of the renewal density have been established. It will be seen that they are substantially less restrictive than the sufficient conditions of Smith [7]; for example, no restraint is placed on the behavior of $f(x)$ for negative $x$.

Condition (ii) of Theorem 1 appears to "beg the question" and does not not seem easy to verify in any particular case; however, the following theorem enables one to recognize quickly a wide class of functions which satisfy this condition.

Theorem 2. Suppose that, for some $\delta>0$, 


$$
\int_{0}^{\delta} b_{\delta}(x) d x<1
$$

and $b_{\delta}(x)=w_{1}(x)+w_{2}(x)$, where $w_{1}(x)$ and $w_{2}(x)$ are non-negative functions which vanish identically outside the interval $(0, \delta)$ and are such that

(i) $w_{1}^{\dagger}(\theta) \in L_{p}$ for some $p>0$;

(ii) $w_{2}(x)=O\left(x^{-M}\right)$ for some $M>0$; then we may conclude that

$$
\Delta\left[b_{\delta}(x)\right] \rightarrow 0, \text { as } x \rightarrow \infty .
$$

From this theorem we shall deduce a simple corollary which states that $\Delta\left[b_{\delta}(x)\right] \rightarrow 0$ as $x \rightarrow \infty$ if $f(x)$ is monotone decreasing in the interval $(0, \delta)$.

The motivation behind the conditions of Theorem 1 can be appreciated intuitively. Only singularities of $f(x)$ in the open interval $(0, \infty)$ should affect the behavior of $\Delta[f(x)]$ for very large $x$. Singularities, if any, of $f(x)$ at the origin should remain at the origin for $f^{* n}(x), n>1$, and not be displaced to larger values of $x$. Thus, for large values of $x, \Delta[f(x)]$ should not be affected by singularities of $f(x)$ at the origin. Needless to say, the "counter-example" from the theory of dams, which we quoted above, satisfies the new set of conditions.

The question arises as to whether there exist probability density functions which fail to satisfy any of the conditions of Theorem 1. We shall show that indeed there are. In addition we shall show that if condition (iii) of Theorem 1 is not satisfied then there exists no large number $\gamma$ such that $\Delta[f(x)]$ is bounded for $x>\gamma$. Concerning condition (ii) of Theorem 1 we shall prove

Theorem 3. If there is some interval $(\alpha, \beta), 0 \leqq \alpha<\beta$, within which $\Delta[f(x)]$ is bounded, then, for all sufficiently small $\delta>0, \Delta\left[b_{\delta}(x)\right] \rightarrow 0$ as $x \rightarrow \infty$.

Thus, when condition (ii) of Theorem 1 fails to hold for any $\delta>0$, the renewal density is unbounded in every interval, however small, to the right of the origin. On the other hand, a Corollary will show that if $\Delta\left[b_{\delta}(x)\right]$ tends to zero as $x \rightarrow \infty$ then it does so exponentially rapidly.

Let $\mathcal{F}$ be the class of density functions $f(x)$ which satisfy the conditions of Theorem 1. It is of some interest to discover closure properties of $F$. In this connection we shall prove

TheOREM 4. Let $f_{1}(x), f_{2}(x)$ belong to $F$; let $p, q$ be non-negative constants, $p+q=1$; let $\phi(x)$ be a bounded non-negative function such that

$$
\int_{-\infty}^{+\infty} \phi(x) f_{1}(x) d x>0 ; \quad \int_{-\infty}^{+\infty} x \phi(x) f_{1}(x) d x>0 .
$$

Then the following probability density functions also belong to F: 


$$
\begin{gathered}
\phi(x) f_{1}(x) /\left\{\int_{-\infty}^{+\infty} \phi(x) f_{1}(x) d x\right\}, \\
p f_{1}(x)+q f_{2}(x), \\
\int_{-\infty}^{+\infty} f_{1}(x-z) f_{2}(z) d z .
\end{gathered}
$$

Finally we shall discuss one or two minor matters, such as the behavior of $\Delta[f(x)]$ as $x \rightarrow-\infty$, and the implications of a recent paper by Feller and Orey [4].

2. Preliminaries. In all that follows $f(x)$ will always stand for the probability density function of a random variable $X$ with strictly positive (possibly infinite) mean value. This assumption will be implicit even when not explicitly invoked. Note that there is no restriction to strictly non-negative random variables. We shall always denote $\varepsilon X$ by $\mu_{1}$, with the understanding, sometimes tacit, that $\mu_{1}^{-1}$ is to be interpreted as zero if $\mu_{1}=\infty$. Our arguments will involve several manipulations of multiple, especially double, series. We shall indulge in these manipulations without comment. The justification will always be the non-negativity of the terms involved. Nearly every statement, relation, or equation, in this paper is really only true (or proven) for almost all $x$; to avoid tiresome repetition, however, we shall always omit mention of this "almost everywhere" qualification.

We shall be primarily concerned with non-negative functions in $L_{1}(-\infty,+\infty)$ and it is convenient to give this class the name $P$. We shall always write $h(x)$ for the renewal density function $\Delta[f(x)] ; m, n, r, s$ and $N$ will denote integers (frequently they are dummy variables in summation); subject to the preceding exceptions, however, we use lower case italic letters for non-negative functions of the variable $x$, and usually these functions are in $P$.

If $a$ and $b$ are non-negative functions then the convolution

$$
\int_{-\infty}^{+\infty} a(x-z) b(z) d z
$$

will always be defined (though possibly infinite). As such convolutions will occur very often in what follows we shall denote them simply as $a * b$ or $a * b(x)$, whichever is the more convenient in a given context. Notice that $a * b=b * a$, and that, whenever the Fourier transforms exist, $(a * b) \dagger=a^{\dagger} b^{\dagger}$.

If $k$ is any non-negative function we shall write $k^{* 2}$ for $k * k$ and, more generally, $k^{*_{n}}$ for $k * k^{*(n-1)}$. Thus, as explained in the introduction, if we define $k^{* 1}=k$ then we shall write

$$
\Delta[k] \equiv \sum_{n=1}^{\infty} k^{* n}
$$


It is clear that the function $\Delta[k]$ is defined almost everywhere, since all the convolutions $k^{*_{n}}$ are non-negative.

We shall also write $\|k\|$ for the integral

$$
\int_{-\infty}^{+\infty} k(x) d x
$$

3. The basic lemmas. In this section we prove the basic lemmas on which our main argument depends.

Lemma 1. (i) If $a$ and $b$ are both in $\odot$ then $a * b$ is in $P$ and $\|a * b\|=\|a\|\|b\|$.

(ii) If $a$ is in $\odot$ and $\|a\|<1$ then $\Delta[a]$ is in $P$ and $\|\Delta[a]\|=\|a\| /(1-\|a\|)$.

Proof. (i) The fact that $a * b$ is non-negative is trivial; and the fact that $a * b$ is in $L_{1}$ is a familiar property of the convolution of two $L_{1}$-functions. That $\|a * b\|=\|a\|\|b\|$ can be shown by direct integration.

(ii) Since $a$ is non-negative it is trivial that $a^{* n}$ is likewise, for $n=2,3, \cdots$, and hence that $\Delta[a]$ is non-negative; moreover, this nonnegativity justifies term-by-term integration, thus

$$
\|\Delta[a]\|=\sum_{n=1}^{\infty}\left\|a^{* n}\right\|
$$

But (i) demonstrates that $\left\|a^{* n}\right\|=\|a\|^{n}$ and so Lemma 1(ii) follows upon summing a geometric series.

We now define a subclass $\mathscr{H}$ of $\mathcal{P}$ which will play an important role in our arguments. The function $a(x) \in \mathscr{N}$ if and only if $a(x) \in \mathcal{P}$ and $a(x) \rightarrow 0$ as $x \rightarrow \infty$. Concerning this class $\mathfrak{N}$ we have the following lemma which shows that $\mathfrak{N}$ is closed under additions, convolutions, and multiplication by nonnegative constants.

Lemma 2. (i) If $k \in \mathfrak{N}$ and $\lambda$ is any non-negative number then $\lambda k \in \mathfrak{N}$.

(ii) If $k_{1}$ and $k_{2}$ are both members of $\mathfrak{N}$ then both $k_{1}+k_{2}$ and $k_{1} * k_{2}$ are members of $\Re$.

Proof. The only nontrivial part of this lemma is the fact that $k_{1} * k_{2} \in \Re$ if both $k_{1} \in \mathscr{N}$ and $k_{2} \in \mathscr{N}$. To prove this point we observe first that $k_{1} * k_{2} \in \mathcal{P}$ by Lemma 1 (i). Furthermore, given any $\epsilon>0$, we can find a large $x_{0}(\epsilon)$ such that both $k_{1}(x)<\epsilon$ and $k_{2}(x)<\epsilon$ for all $x>x_{0}$. If, therefore, we choose $x>2 x_{0}$ it follows that

$$
\begin{aligned}
k_{1} * k_{2}(x) & =\int_{-\infty}^{x / 2}+\int_{x / 2}^{+\infty} k_{1}(x-z) k_{2}(z) d z \\
& <\epsilon\left\|k_{1}\right\|+\epsilon\left\|k_{2}\right\| .
\end{aligned}
$$

Plainly, this implies that $k_{1} * k_{2}(x) \rightarrow 0$ as $x \rightarrow \infty$ and the lemma is proved. 
The mutilations $a(x), b_{\delta}(x), c_{\delta}(x)$ of $f(x)$ have been defined in our introduction. We next prove a lemma involving $b_{\delta}(x)$.

LEMMA 3. If $\Delta\left[b_{\delta}\right] \in \Re$ and $\lambda$ is any real non-negative number then there is a $\delta^{*}>0$ such that $\Delta\left[\lambda b_{\delta^{*}}\right] \in \Re$.

Proof. Since $\Delta\left[b_{0}\right] \in \mathcal{N}$ there must be an $x_{0}(\epsilon)$ such that $\Delta\left[b_{\delta}(x)\right]<\epsilon$ for all $x>x_{0}$. In particular, this implies that $b_{\delta}^{* n}(x)<\epsilon$ for all $x>x_{0}$ and all $n$. But

$$
\begin{aligned}
b_{\delta}^{* 2 n}(x) & =\int_{0}^{x / 2}+\int_{x / 2}^{x} b_{\delta}^{* n}(x-z) b_{\delta}^{* n}(z) d z \\
& =2 \int_{x / 2}^{x} b_{\delta}^{* n}(x-z) b_{\delta}^{* n}(z) d z
\end{aligned}
$$

and so, for all $x>2 x_{0}(\epsilon)$,

$$
b_{\delta}^{* 2 n}(x) \leqq 2 \epsilon\left\|b_{\delta}^{* n}\right\|=2 \epsilon\left\|b_{\delta}\right\|^{n} .
$$

We choose $\delta^{*} \leqq \delta$ such that $4 \lambda^{2}\left\|b_{\delta^{*}}\right\|<1$. Then, necessarily, $\Delta\left[b_{\delta^{*}}\right] \leqq \Delta\left[b_{\delta}\right]$ and hence $\Delta\left[b_{\delta^{*}}\right] \in \mathfrak{N}$ also. Thus the preceding argument on $b_{\delta}$ applies equally well to $b_{\delta^{*}}$. Therefore, for all $x>2 x_{0}(\epsilon)$,

and so

$$
\lambda^{2 n} b_{\delta^{*}}^{* 2 n}(x)<2 \epsilon\left(\frac{1}{4}\right)^{n}
$$

$$
\sum_{n=1}^{\infty} \lambda^{2 n} b_{\delta^{*}}^{* 2 n}(x)<\epsilon
$$

Similarly, one can show that

$$
\sum_{n=1}^{\infty} \lambda^{2 n-1} b_{\delta^{*}}^{*(2 n-1)}(x)<\epsilon
$$

for all sufficiently large $x$, and a combination of the last two inequalities proves the lemma.

With the aid of Lemma 3 we are able to prove the following lemma which we shall use directly in the proof of our main theorem.

LEMma 4. If $\Delta\left[b_{8}\right] \in \mathscr{N}$ then there is $a \delta^{*}>0$ such that $\Delta\left[a+b_{\delta^{*}}\right] \in \Re$.

Proof. Note that $\|a\|<1$ so that we may choose a $\lambda$ such that $0<\lambda<1$ - $\|a\|$. Then, by Lemma 3 , we can find a $\delta^{*}, 0<\delta^{*} \leqq \delta$, such that

$$
\Delta\left[\lambda^{-1} b_{\delta^{*}}\right]=\sum_{n=1}^{\infty} \lambda^{-n} b_{\delta^{*}}^{* n}
$$

belongs to $\Re ; \Delta\left[b_{\delta^{*}}\right]$, of course, also belongs to $\Re$.

For each $n \geqq 0$ let us define the non-negative function 


$$
u_{n}(x)=\sum_{m=0}^{\infty}\left(\begin{array}{c}
n+m \\
m
\end{array}\right)\left(a+b_{\delta^{*}}\right) * a^{* m}
$$

Evidently,

$$
\begin{aligned}
\left\|u_{n}\right\| & =\sum_{m=0}^{\infty}\left(\begin{array}{c}
n+m \\
m
\end{array}\right)\left(\|a\|+\left\|b_{\delta^{*}}\right\|\right)\|a\| m \\
& =\frac{\|a\|+\left\|b_{\delta^{*}}\right\|}{(1-\|a\|)^{n+1}}
\end{aligned}
$$

and so $u_{n}(x)$ belongs to $P$. Furthermore, since $a(x)$ vanishes identically for $x \geqq 0$, it is apparent from the definition of $u_{n}(x)$ that $u_{n}(x)$ vanishes identically for $x \geqq \delta^{*}$.

Next define

$$
u(x)=\sum_{n=1}^{\infty} \lambda^{n} u_{n}(x),
$$

where $\lambda$ is the number chosen above. Plainly $u(x)$ is non-negative and vanishes identically for $x \geqq \delta^{*}$. Moreover,

$$
\|u\|=\sum_{n=1}^{\infty} \lambda^{n} \frac{\left(\|a\|+\left\|b_{\delta^{*}}\right\|\right)}{(1-\|a\|)^{n+1}}
$$

and so, by our choice of $\lambda, u \in L_{1}$, and hence $u \in \Re$. We have therefore shown that both $\Delta\left[\lambda^{-1} b_{\gamma^{*}}\right]$ and $u$ belong to $\Re$ and may appeal to Lemma 2 (ii) to infer that $u * \Delta\left[\lambda^{-1} b_{\delta^{*}}\right]$ also belongs to $\Re$. But

$$
0 \leqq \sum_{n=1}^{\infty}\left(\lambda^{n} u_{n}\right) *\left(\lambda^{-n} b_{\partial^{*}}^{* n}\right) \leqq u * \Delta\left[\lambda^{-1} b_{\delta^{*}}\right]
$$

since the summation of this inequality involves only the "diagonal" terms that arise in the calculation of $u * \Delta\left[\lambda^{-1} b_{\delta^{*}}\right]$. Thus $\sum_{1}^{\infty} u_{n} * b_{\delta^{*}}^{* n}$ and, hence, $u_{0}+\sum_{1}^{\infty} u_{n} * b_{\delta^{*}}^{* n}$ belong to $\Re$. However,

$$
\begin{aligned}
u_{0}+\sum_{n=1}^{\infty} u_{n} * b_{\delta^{*}}^{* n} & =\sum_{m=0}^{\infty}\left(a+b_{\delta^{*}}\right) * a^{* m}+\sum_{n=1}^{\infty} \sum_{m=0}^{\infty}\left(\begin{array}{c}
n+m \\
m
\end{array}\right)\left(a+b_{\delta^{*}}\right) * a^{* m} * b_{\delta^{*}}^{* n} \\
& =\sum_{m=0}^{\infty} \sum_{n=0}^{\infty}\left(\begin{array}{c}
n+m \\
m
\end{array}\right)\left(a+b_{\delta^{*}}\right) * a^{* m} * b_{\delta^{*}}^{* n} \\
& =\sum_{k=0}^{\infty} \sum_{r+s=k}\left(\begin{array}{c}
r+s \\
r
\end{array}\right)\left(a+b_{\delta^{*}}\right) * a^{* n} * b_{\delta^{*}}^{* n} \\
& =\sum_{k=0}^{\infty}\left(a+b_{\delta^{*}}\right)^{*(k+1)} \\
& =\Delta\left[a+b_{\delta^{*}}\right]
\end{aligned}
$$


This proves the lemma. We note, however, that the final algebraic steps could be considerably simplified if $a$ and $b_{\delta^{*}}$ were manipulated as though they were real numbers, instead of functions forming convolutions. It is necessary, nevertheless, to demonstrate that the desired result can be achieved by legitimate operations on functions, and not by purely "symbolic" operations.

LEMMA 5. $\theta^{2} /\left\{1-f^{\dagger}(\theta)\right\}$ is bounded in a neighborhood of 0 .

Proof. It is easy to see that

$$
\left|\frac{1-f^{\dagger}(\theta)}{\theta^{2}}\right| \geqq \int_{-\infty}^{+\infty} \frac{(1-\cos \theta x)}{\theta^{2}} f(x) d x,
$$

and, since the integrand on the right of this inequality is non-negative, we have that for any large positive $\gamma$,

$$
\left|\frac{1-f^{\dagger}(\theta)}{\theta^{2}}\right| \geqq \int_{-\gamma}^{+\gamma} \frac{1-\cos \theta x}{\theta^{2}} f(x) d x .
$$

But, given any small $\epsilon>0$ we can find a $\delta(\epsilon)$ such that $(1-\cos y)$ $>\frac{1}{2}(1-\epsilon) y^{2}$ for all $|y|<\delta$. Hence, if we choose $|\theta|<\delta / \gamma$,

$$
\left|\frac{1-f^{\dagger}(\theta)}{\theta^{2}}\right| \geqq \frac{1}{2}(1-\epsilon) \int_{-\gamma}^{+\gamma} x^{2} f(x) d x .
$$

By choosing $\gamma$ large enough we can always make

$$
\int_{-\gamma}^{+\gamma} x^{2} f(x) d x>0
$$

and so the lemma is proved.

As a matter of fact, we only strictly need in the sequel the weaker result that $\theta^{2} /\left\{1-f^{\dagger}(\theta)\right\}$ is integrable in a neighborhood of the origin; this weaker result can be inferred from the general Lemma 8 of Smith [9]. A desire for completeness, and the simplicity of the above proof, suggest we give it here.

LEMMA 6.

$$
\frac{1-\frac{\sin (\theta / 2)}{\theta / 2}}{1-f^{\dagger}(\theta)}
$$

is a bounded function of $\theta$.

Proof. By a well-known property of the characteristic function of an absolutely continuous distribution, given any $\epsilon>0$ there is an $\eta(\epsilon)>0$ such that $\left|f^{\dagger}(\theta)\right| \leqq 1-\eta$ for all $|\theta| \geqq \epsilon$. Hence, for all $|\theta| \geqq \epsilon$, 


$$
\left|\frac{1-\frac{\sin (\theta / 2)}{\theta / 2}}{1-f^{\dagger}(\theta)}\right| \leqq \frac{2}{\eta} .
$$

But, for $|\theta|<\epsilon$, it is easily shown that

$$
1-\frac{\sin (\theta / 2)}{\theta / 2}=O\left(\theta^{2}\right)
$$

Thus the lemma follows from an appeal to Lemma 5 .

Lemma 7. If the function $k(x)$ belongs to $P$ and its transform $k^{\dagger}(\theta)$ belongs to $L_{1}$ then

$$
k * h(x) \rightarrow \frac{\|k\|}{\mu_{1}}
$$

as $x \rightarrow \infty\left(\mu_{1}^{-1}\right.$ is defined to mean zero when $\left.\mu_{1}=+\infty\right)$.

Proof. We may suppose $\|k\|>0$ since otherwise the lemma is trivial. Since $k^{\dagger}(\theta) \in L_{1}$ we have (Theorem 8 of Bochner and Chandrasekharan [2]) that

$$
k(x)=\frac{1}{2 \pi} \int_{-\infty}^{+\infty} e^{-i \theta x} k^{\dagger}(\theta) d \theta .
$$

Thus $|k(x)|$ is bounded, by $(2 \pi)^{-1}\left\|k^{\dagger}\right\|$, and the Riemann-Lebesgue lemma shows that $k(x) \rightarrow 0$ as $|x| \rightarrow \infty$.

Let us next write

$$
u_{N}(x)=\sum_{n=1}^{N} k * f^{* n}
$$

Then, because all terms involved are non-negative, we can introduce the limit

$$
u_{\infty}(x)=\lim _{N \rightarrow \infty} u_{N}(x)=k * h(x)
$$

Furthermore,

$$
u_{N}^{\dagger}(\theta)=\sum_{n=1}^{N} k^{\dagger}\left(f^{\dagger}\right)^{n}
$$

and so it is plain that $u_{N}^{\dagger}$ belongs to $L_{1}$ and that we may write

$$
u_{N}(x)=\frac{1}{2 \pi} \int_{-\infty}^{+\infty} e^{-i \theta x} k^{\dagger}(\theta) f^{\dagger}(\theta)\left\{\frac{1-\left(f^{\dagger}(\theta)\right)^{N+1}}{1-f^{\dagger}(\theta)}\right\} d \theta .
$$


Next define

$$
v_{N}(x)=\int_{x-1 / 2}^{x+1 / 2} u_{N}(z) d z
$$

and note that, by the monotone convergence theorem, we can write

$$
v_{\infty}(x)=\lim _{N \rightarrow \infty} v_{N}(x)=\int_{x-1 / 2}^{x+1 / 2} u_{\infty}(z) d z .
$$

Routine calculation shows that

$$
\stackrel{+}{v_{N}(\theta)}=\frac{\sin (\theta / 2)}{(\theta / 2)} u_{N}^{\dagger}(\theta),
$$

so that, since $(\sin (\theta / 2)) /(\theta / 2)$ is a bounded function of $\theta, v_{N}^{\dagger}(\theta)$ is also in $L_{1}$, like $u_{N}(\theta)$. Thus

$$
u_{N}(x)-v_{N}(x)=\frac{1}{2 \pi} \int_{-\infty}^{+\infty} e^{-i \theta x} k^{\dagger}(\theta) f^{\dagger}(\theta)\left\{\frac{1-\frac{\sin (\theta / 2)}{\theta / 2}}{1-f^{\dagger}(\theta)}\right\}\left\{1-\left(f^{\dagger}(\theta)\right)^{N+1}\right\} d \theta .
$$

If we now appeal to Lemma 6 and use the fact that $|f t(\theta)| \leqq 1$ it is clearly seen that the integrand on the right is dominated by some multiple of the integrable function $\left|k^{\dagger}(\theta)\right|$. Moreover, $\left|f^{\dagger}(\theta)\right|<1$ for all $\theta \neq 0$ so that $\left\{f^{\dagger}(\theta)\right\}^{N}$ $\rightarrow 0$ as $N \rightarrow \infty$ for all $\theta \neq 0$. Thus we can appeal to Lebesgue's theorem on dominated convergence to deduce that

$$
u_{\infty}(x)-v_{\infty}(x)=\frac{1}{2 \pi} \int_{-\infty}^{+\infty} e^{-i \theta x} k^{\dagger}(\theta) f^{\dagger}(\theta)\left\{\frac{1-\frac{\sin (\theta / 2)}{\theta / 2}}{1-f^{\dagger}(\theta)}\right\} d \theta .
$$

However, the integrand on the right of this last equation is also dominated by some multiple of $\left|k^{\dagger}(\theta)\right|$ and so belongs to $L_{1}$. Thus we can effect a further appeal to the Riemann-Lebesgue lemma to infer that

$$
u_{\infty}(x)-v_{\infty}(x) \rightarrow 0
$$

as $|x| \rightarrow \infty$. Moreover, since $u_{\infty}=k * h$, it follows that, as $|x| \rightarrow \infty$,

$$
k * h(x)-\int_{x-1 / 2}^{x+1 / 2} k * h(z) d z \rightarrow 0
$$

The lemma will therefore be proved if we can show that, as $x \rightarrow \infty$,

$$
\int_{x-1 / 2}^{x+1 / 2} k * h(z) d z \rightarrow \mu_{1}^{-1}\|k\| .
$$


To this end, let us put

$$
g(x)=\int_{x-1 / 2}^{x+1 / 2} h(z) d z .
$$

Then $g(x)$ can be interpreted as the expected number of renewals to occur in the interval $(x-1 / 2, x+1 / 2)$, and we can deduce from renewal theory that $g(x)$ is bounded and that, by Blackwell's theorem, $g(x) \rightarrow \mu_{1}^{-1}$ as $x \rightarrow \infty$ (Blackwell [1]). We can then appeal to a very easily proved lemma of Smith $\left[6\right.$, Lemma 1] to infer that $k * g(x) \rightarrow \mu_{1}^{-1}\|k\|$ as $x \rightarrow \infty$. But routine computation will show that

$$
k * g(x)=\int_{x-1 / 2}^{x+1 / 2} k * h(z) d z,
$$

and this completes the proof.

LEMMA 8. If $u$ and $v$ are both members of $P$ and if we write

then, for any integer $N$,

$$
\begin{aligned}
k & =v+v * \Delta[u], \\
l & =k+\Delta[u],
\end{aligned}
$$

$$
\Delta[u+v]=l+l * k+l * k^{* 2}+\cdots+l * k^{*(N-1)}+k^{* N} * \Delta[u+v] .
$$

Proof. Like part of the proof of Lemma 4 this lemma is easily seen if we treat $u, v, l, k$ as reals; but it is again, of course, desirable to establish the result by justifiable operations.

We note first that

$$
\begin{aligned}
l+k * \Delta[u+v]= & v+\sum_{n=1}^{\infty} u^{* n}+\sum_{n=1}^{\infty} v * u^{* n}+\sum_{n=1}^{\infty} v *(u+v)^{* n} \\
& +\sum_{m=1}^{\infty} \sum_{n=1}^{\infty} v * u^{* m} *(u+v)^{* n} \\
= & \sum_{n=1}^{\infty} u^{* n}+\sum_{m=0}^{\infty} \sum_{n=0}^{\infty} v * u^{* m} *(u+v)^{* n} \\
= & \sum_{n=1}^{\infty} u^{* n}+\sum_{m=0}^{\infty} \sum_{n=0}^{\infty} \sum_{r=0}^{n}\left(\begin{array}{c}
n \\
r
\end{array}\right) u^{*(m+r) * v^{*(n+1-r)} .}
\end{aligned}
$$

For any integers $\alpha, \beta, \alpha \geqq 0, \beta \geqq 1$, the term $u^{* \alpha * v * \beta}$ can arise in the triple summation on the right in $(1+\alpha)$ ways, corresponding to $r=0,1,2, \cdots, \alpha$. The total coefficient of $u^{* \alpha * v}{ }^{* \beta}$ turns out to be

$$
\left(\begin{array}{c}
\beta-1 \\
0
\end{array}\right)+\left(\begin{array}{l}
\beta \\
1
\end{array}\right)+\cdots+\left(\begin{array}{c}
\alpha+\beta-1 \\
\alpha
\end{array}\right)
$$


and a simple inductive argument proves this to equal

$$
\left(\begin{array}{c}
\alpha+\beta \\
\beta
\end{array}\right) \text {. }
$$

Thus

$$
\begin{aligned}
l+k * \Delta[u+v] & =\sum_{n=1}^{\infty} u^{* n}+\sum_{\alpha=0}^{\infty} \sum_{\beta=1}^{\infty}\left(\begin{array}{c}
\alpha+\beta \\
\alpha
\end{array}\right) u^{* \alpha} * v^{* \beta} \\
& =\sum_{n=1}^{\infty} \sum_{\alpha+\beta=n}\left(\begin{array}{l}
n \\
\alpha
\end{array}\right) u^{* \alpha} * v^{* \beta} \\
& =\sum_{n=1}^{\infty}(u+v)^{* n} \\
& =\Delta[u+v] .
\end{aligned}
$$

We have thus proved that the lemma holds for $N=1$, and the general result follows by repeated applications of the special result. For example,

$$
\begin{aligned}
\Delta[u+v] & =l+k *(l+k * \Delta[u+v]) \\
& =l+k * l+k^{* 2} * \Delta[u+v]
\end{aligned}
$$

and so on.

Lemma 9. If $h(x) \rightarrow \mu_{1}^{-1}$ as $x \rightarrow \infty$ and if $k(x)$ is any function in $p$ then $\lim \inf _{x \rightarrow \infty} k * h(x) \geqq \mu_{i}^{-1}\|k\|$.

Proof. The lemma follows immediately from Fatou's Lemma, since

$$
k * h(x)=\int_{-\infty}^{+\infty} h(x-z) k(z) d z,
$$

$h(x-z) k(z) \geqq 0$, and $h(x-z) k(z) \rightarrow \mu_{1}^{-1} k(z)$ for all $z$ as $x \rightarrow \infty$.

4. Proof of Theorem 1. We deal with the necessity part first.

The proof that condition (i) must necessarily hold has been attributed to Feller [3], but we have recently noticed that Feller's proof needs to assume the boundedness of $h(x)$; most of our difficulties in the present investigation arise precisely because we may not assume $h(x)$ to be bounded. However, the necessity of (i) is easy to prove by an appeal to Lemma 9 . We observe that $h(x)$ satisfies the familiar integral equation

$$
h(x)=f(x)+f * h(x)
$$

and so, if $h(x) \rightarrow \mu_{1}^{-1}$ as $x \rightarrow \infty$, it follows that

$$
\limsup _{x \rightarrow \infty} f(x)=\mu_{1}^{-1}-\liminf _{x \rightarrow \infty} f * h(x) .
$$

An appeal to Lemma 9 then shows that we must have $f(x) \rightarrow 0$. 
Next, to prove the necessity of (ii), choose any $\delta>0$ such that $\left\|b_{b}\right\|<1$. We appeal to Lemma 8, making in it the substitutions $N=1, u=b_{\delta}, v=a+c_{8}$. This yields

$$
\Delta[f]=v+v * \Delta[u]+\Delta[u]+k * \Delta[f],
$$

where

$$
\begin{aligned}
k & =v+v * \Delta[u], \\
& =\left(a+c_{\delta}\right)+\left(a+c_{\delta}\right) * \Delta\left[b_{\delta}\right] .
\end{aligned}
$$

The functions $u(x)$ and $v(x)$, as presently defined, are clearly in the class 8 . Thus, by an appeal to Lemma 1 it follows that $k(x)$ is also in $\mathcal{P}$ and that

$$
\|k\|=\left\{\|a\|+\left\|c_{\delta}\right\|\right\}\left\{1+\frac{\left\|b_{\delta}\right\|}{1-\left\|b_{\delta}\right\|}\right\}=1,
$$

since $\|a\|+\left\|b_{\delta}\right\|+\left\|c_{\delta}\right\|=\|f\|=1$.

We are at the moment assuming that $h(x) \rightarrow \mu_{1}^{-1}$ as $x \rightarrow \infty$, and so it follows from the properties just deduced for $k(x)$ and from Lemma 9 that

$$
\liminf _{x \rightarrow \infty} k * \Delta[f] \geqq \mu_{1}^{-1} .
$$

Therefore, from (4.1) we infer that

$$
\limsup _{x \rightarrow \infty} v(x)+v * \Delta[u(x)]+\Delta[u(x)] \leqq 0 .
$$

The non-negativity of all the terms involved in the last formula allows the deduction that $\Delta[u] \equiv \Delta\left[b_{\delta}\right] \rightarrow 0$ as $x \rightarrow \infty$, and thereby proves the necessity of (ii).

To conclude the necessity part of the proof we deal with (iii). We begin by observing that if $h(x) \rightarrow \mu_{1}^{-1}$ as $x \rightarrow \infty$ then there must be some $\gamma$ such that $h(x)<1$ for all $x \geqq \gamma$. However, it is trivial that $h(x) \geqq c_{\delta}^{* n}(x)$ for all $\delta>0$ and all $n$. In particular, this last inequality will hold if we take $n=m$, say, where $m$ is the next integer greater than $\gamma / \delta$. Thus $c_{\delta}^{* m}(x)$ is bounded for all $x \geqq \gamma$. But it is not difficult to see that, because of our choice of $m, c_{\delta}^{* m}(x)$ vanishes identically for $x<\gamma$. Thus $c_{\delta}^{* m}(x)$ is bounded everywhere and, being already known to be a member of $L_{1}$, it must also be a member of $L_{2}$. By the reciprocity of $L_{2}$ Fourier transforms it then follows that $\left(c_{\delta}^{\dagger}(\theta)\right)^{m}$, the Fourier transform of $c_{\delta}^{* m}(x)$, is in $L_{2}$; this shows that $c_{\delta}^{\dagger}(\theta)$ is a member of $L_{p}$ with $p=2 m$. The necessity of (iii) is thus established.

Let us now assume that, for some $\delta>0$, the conditions (i), (ii), and (iii) hold, and prove them sufficient to make $h(x) \rightarrow \mu_{1}^{-1}$ as $x \rightarrow \infty$.

Suppose first that $\left\|b_{\delta}\right\|=1$. Then $b_{\delta}=f$ and we see from (ii) that $h(x)$ $=\Delta\left[b_{\delta}(x)\right] \rightarrow 0$ as $x \rightarrow \infty$. It follows easily from this result that if $k(x)$ is any bounded function in $\mathcal{P}$ then $k * h(x) \rightarrow 0$ as $x \rightarrow \infty$. Comparison with Lemma 7 
then shows that we must have $\mu_{1}=\infty$; but this is impossible, since $f$ vanishes outside the interval $(0, \delta)$. Thus it must be that $\left\|b_{\delta}\right\|<1$.

Next we shall show that, with no loss of generality, it may be supposed that $\left(a+b_{\delta}\right) \in \mathcal{X}$ and that $\left\|c_{\delta}\right\|>0$. For, since it is given that $\Delta\left[b_{\delta}(x)\right] \rightarrow 0$ as $x \rightarrow \infty$, and since we must have $\left\|b_{\delta}\right\|<1$, it is an easy matter to show that $\Delta\left[b_{\delta}\right] \in \Re$. Then, by Lemma 4 , we can choose $\delta^{*}>0$ such that $\Delta\left[a+b_{\delta^{*}}\right] \in \Re$, and this $\delta^{*}$ can be chosen small enough to make $\left\|c_{\delta^{*}}\right\|>0$. Now the necessity arguments we applied above to $\Delta[f]$ work equally well upon $\Delta\left[b_{\delta}\right]$, since we are assuming $\Delta\left[b_{\delta}(x)\right]$ tends to a limit as $x \rightarrow \infty$, and they allow us to infer that $\left(b_{\delta}-b_{\delta^{*}}\right) \dagger$ belongs to some class $L_{q}$. But we are given in condition (iii) that $c_{\delta}^{\dagger}$ belongs to $L_{p}$. In view of the fact that both $\left(b_{\delta}-b_{\delta^{*}}\right) \dagger$ and $c_{\delta}^{\dagger}$ are necessarily bounded functions of $\theta$ it is evident that they both belong to $L_{r}$, where $r=\operatorname{Max}(p, q)$. Thus, by a familiar application of Minkowski's inequality, $c_{\delta}^{\dagger}=c_{\delta}^{\dagger}+\left(b_{\delta}-b_{\delta^{*}}\right) \dagger$ is also in $L_{r}$ (we may assume $r>1$ ). Thus the conditions (ii) and (iii) hold with $\delta$ replaced by $\delta^{*}$, and, in addition, we have that $\left(a+b_{\delta^{*}}\right) \in \Re$ and that $\left\|c_{\delta^{*}}\right\|>0$. This completes our justification of the claim made at the start of this paragraph.

We shall make use in our argument of the functional identity proved in Lemma 8 . In this lemma we take $N$ as any integer greater than $p$ and let $u=a+b_{\delta}$ and $v=c_{\delta}$. Then, in the notation of Lemma 8 ,

$$
k=c_{\delta}+c_{\delta} * \Delta\left[a+b_{\delta}\right]
$$

and it is easy to deduce from Lemma 1 that $k$ is in $P$. Moreover as $M \rightarrow \infty$, the function

$$
c_{\delta}+\sum_{n=1}^{M} c_{\delta} *\left(a+b_{\delta}\right)^{* n}
$$

increases to the $L_{1}$ limit-function $k$; thus it is a simple matter to justify the following term-by-term calculation of $k^{\dagger}$;

$$
\begin{aligned}
k^{\dagger} & =c_{\delta}^{\dagger}+\sum_{n=1}^{\infty} c_{\delta}^{\dagger}\left(a^{\dagger}+b_{\delta}^{\dagger}\right)^{n} \\
& =\frac{c_{\delta}^{\dagger}}{1-\left(a^{\dagger}+b_{\delta}^{\dagger}\right)} .
\end{aligned}
$$

Therefore, if we use the fact that $\left|a^{\dagger}+b_{\delta}^{\dagger}\right| \leqq\|a\|+\left\|b_{8}\right\|=1-\left\|c_{8}\right\|<1$, we can deduce that

$$
\begin{aligned}
\left|k^{\dagger}\right| & \leqq \frac{1}{1-\left(\|a\|+\left\|b_{\delta}\right\|\right)}\left|c_{\delta}^{\dagger}\right| \\
& \leqq\left|\begin{array}{c}
\dagger \\
c_{\delta}
\end{array}\right| /\left\|c_{\delta}\right\| .
\end{aligned}
$$

But $\left|c_{\delta}^{\dagger}\right| \leqq\left\|c_{\delta}\right\|$ so that it is obvious now that $\left|k^{\dagger}\right|$ belongs to $L_{p}$ and is bounded by unity. Thus $\left|k^{\dagger}\right|^{N}$ belongs to $L_{1}$, and we can appeal to Lemma 7 
to infer that, as $x \rightarrow \infty$,

$$
k^{* N} * h(x) \rightarrow \mu_{1}^{-1}\|k\|^{N} .
$$

However, it can be shown by using Lemma 1 , that

$$
\|k\|=\left\|c_{\delta}\right\|\left\{1+\frac{\left\|a+b_{\delta}\right\|}{1-\left\|a+b_{\delta}\right\|}\right\}=1,
$$

so that (4.3) can actually be written more simply as

$$
k^{* N} * h(x) \rightarrow \mu_{1}^{-1} \text {. }
$$

Let us return to (4.2). We have shown that $\Delta\left[a+b_{\delta}\right]$ may be assumed in $\Re$ and condition (i) shows that $c_{\delta}$ is in $\Re$. Thus an appeal to Lemma 2 proves $k \in \Re$. Employing further notation from Lemma 8 we have $l=k$ $+\Delta\left[a+b_{\delta}\right]$ is also a number of $\Re$. Thus, by Lemma $2, l+l * k+l * k * 2+\cdots$ $+l * k^{*(N-1)}$ belongs to $\Re$. But Lemma 8 shows that

$$
h(x)=l(x)+l * k(x)+\cdots+l * k^{*(N-1)}(x)+k^{* N} * h(x),
$$

so the theorem is proved by an appeal to (4.4).

5. Proof of Theorem 2. Since $\left|w_{1}^{\dagger}(\theta)\right| \leqq\left\|w_{1}(x)\right\|<1$ we can suppose $p \geqq 2$. Choose $\delta^{*}>0$ so that

$$
\int_{0}^{\delta *} w_{2}(x) d x \leqq \frac{1}{2^{(M+2)}}
$$

and then define

$$
\begin{array}{rlrl}
\bar{w}_{2}(x) & =w_{2}(x) & & \text { if } x \leqq \delta^{*} \\
& =0 & \text { otherwise; } \\
\bar{w}_{1}(x) & =b_{\delta}(x)-\bar{w}_{2}(x) \\
& =w_{1}(x)+\left(w_{2}(x)-\bar{w}_{2}(x)\right) .
\end{array}
$$

It is apparent from the hypothesis that $w_{2}(x)-\bar{w}_{2}(x)$ is a bounded integrable function, and therefore it belongs to $L_{2}$. Thus its transform, $w_{2}^{\dagger}(\theta)$ $-\bar{w}_{2}^{\dagger}(\theta)$, also belongs to $L_{2}$ and, since $\left|w_{2}^{\dagger}(\theta)-\bar{w}_{2}^{\dagger}(\theta)\right| \leqq\left\|w_{2}-\bar{w}_{2}\right\|<1$, it follows that $w_{2}^{\dagger}(\theta)-\bar{w}_{2}^{\dagger}(\theta)$ belongs to $L_{p}$ (recall that $p>2$ ). By Minkowski's inequality the sum of two $L_{p}$-functions is another $L_{p}$ function (for $p>1$ ). Thus $\bar{w}_{1}(x)$ $=w_{1}(x)+\left(w_{2}(x)-\bar{w}_{2}(x)\right)$ is a non-negative function whose Fourier transform is in $L_{p}$. In other words, with no loss of generality, we may suppose it possible to write $b_{\delta}(x)=w_{1}(x)+w_{2}(x)$ where in addition to the properties required of $w_{1}(x)$ and $w_{2}(x)$ in the enunciation of Theorem 2 we also have $\left\|w_{2}\right\| \leqq 2^{-(M+2)}$.

We shall next prove the intermediate result that, for some constant $\gamma$,

$$
w_{2}^{* n}(x) \leqq \frac{\gamma}{2^{n-1} x^{M}}
$$


for all $n$ and all $x>0$.

We may, by hypothesis, choose a $\gamma$ so that $w_{2}(x)<\gamma x^{-M}$ for all $x>0$. Let us make the inductive hypothesis that $(5.1)$ is true for $n=1,2, \cdots, m$. Then

$$
\begin{aligned}
w_{2}^{*(m+1)}(x) & =\int_{0}^{x / 2}+\int_{x / 2}^{x} w_{2}^{* m}(x-z) w_{2}(z) d z \\
& \leqq \frac{\gamma}{2^{m-1}(x / 2)^{M}} \int_{0}^{x / 2} w_{2}(z) d z+\frac{\gamma}{(x / 2)^{M}} \int_{0}^{x / 2} w_{2}^{* m}(z) d z \\
& \leqq \frac{\gamma}{2^{m-1}(x / 2)^{M}}\left\|w_{2}\right\|+\frac{\gamma}{(x / 2)^{M}}\left\|w_{2}^{* m}\right\| .
\end{aligned}
$$

Evidently, by Lemma 1, $\left\|w_{2}^{* m}\right\|=\left\|w_{2}\right\| m^{m}$; and we have shown it possible to suppose with no loss of generality that $\left\|w_{2}\right\|<2^{-(M+2)}$. Thus

$$
w_{2}^{*(m+1)}(x)<\frac{\gamma}{2^{m+1} x^{M}}+\frac{\gamma}{2^{(m M+2 m-N)} x^{M}}
$$

and, since $m M+2 m-M \geqq m+1$ for all $m \geqq 1$, it follows that

$$
w_{2}^{*(m+1)}(x)<\frac{\gamma}{2^{m} x^{M}}
$$

so that the inductive hypothesis is continued. Since this hypothesis is true for $m=1$, the validity of $(5.1)$ is thereby established for all $m$.

Hence,

$$
\Delta\left[w_{2}(x)\right] \leqq \sum_{n=1}^{\infty} \frac{\gamma}{2^{n-1} x^{M}} \leqq \frac{2 \gamma}{x^{M}}
$$

and so $\Delta\left[w_{2}(x)\right] \rightarrow 0$ as $x \rightarrow \infty$.

Let us next choose an integer $N>p$ and appeal to Lemma 8 again, this time with the substitutions $u=w_{2}$ and $v=w_{1}$. Then $k=w_{1}+w_{1} * \Delta\left[w_{2}\right]$ and so, in view of the result just proved, $k \in \Re$. Thence it follows that $l=k+\Delta\left[w_{2}\right]$ also belongs to $\Re$. Thus, by Lemma 2 ,

$$
l(x)+l * k(x)+\cdots+l * k^{*(N-1)}(x) \rightarrow 0,
$$

as $x \rightarrow \infty$. Furthermore, $k^{* N} * \Delta\left[w_{1}+w_{2}\right]$ is an $L_{1}$ function whose Fourier transform can be shown to be dominated by

$$
\left|w_{1}^{\dagger}\right|^{N}\left\{1+\frac{\left\|w_{2}\right\|}{1-\left\|w_{2}\right\|}\right\}^{N}\left\{1+\frac{\left\|b_{\delta}\right\|}{1-\left\|b_{\delta}\right\|}\right\} .
$$

This Fourier transform must therefore belong to $L_{1}$, because of our assumption about $w_{1}^{\dagger}$. We saw at the beginning of the proof of Lemma 7 that a function whose Fourier transform is in $L_{1}$ must be bounded and must tend to 
zero as $x \rightarrow \infty$. Thus $k^{N} * \Delta\left[w_{1}(x)+w_{2}(x)\right] \rightarrow 0$ as $x \rightarrow \infty$; this fact, coupled with (5.2) and Lemma 8, is enough to show that

$$
\Delta\left[b_{\delta}(x)\right] \equiv \Delta\left[w_{1}(x)+w_{2}(x)\right] \rightarrow 0 \quad \text { as } \quad x \rightarrow \infty .
$$

The lemma is therefore proven.

CoRollary 2.1. If, for some $\delta>0$ for which $\left\|b_{\delta}\right\|<1$, the function $f(x)$ is monotone decreasing in $(0, \delta)$ then $\Delta\left[b_{\delta}\right] \in \Re$.

Proof. If $f(x)$ is monotone decreasing in $(0, \delta)$ then we observe that

$$
\sum_{n=1}^{\infty} f\left(\frac{\delta}{2^{n}}\right) \frac{\delta}{2^{n}} \leqq \sum_{n=1}^{\infty} \int_{\delta / 2^{n}}^{\delta / 2^{n-1}} f(x) d x=\int_{0}^{\delta} f(x) d x<\infty,
$$

since $f(x) \in L_{\text {n. }}$. Thus, for some $\gamma>0$ and all $n \geqq 1, f\left(\delta / 2^{n}\right)<2^{n} \gamma / \delta$. Therefore if $x$ is any number in $(0, \delta)$, and we choose the integer $m$ so that $\delta / 2^{m+1}$ $<x \leqq \delta / 2^{m}$, then $f(x) \leqq f\left(\delta / 2^{m+1}\right) \leqq 2^{m+1} \gamma / \delta<2 \gamma / x$. Hence $f(x)=O\left(x^{-1}\right)$ in $(0, \delta)$ and the corollary follows from Theorem 2 .

Notice that if $f(x)$ is monotone increasing in $(0, \delta)$ then $f(x)$ must be bounded in $\left(0, \delta^{*}\right)$ for any $\delta^{*}<\delta$, and it then follows at once from Theorem 2 that $\Delta\left[b_{\delta^{*}}(x)\right] \rightarrow 0$ as $x \rightarrow \infty$.

6. Some examples. It is of interest to consider briefly some cases in which certain of the conditions of Theorem 1 fail to hold.

Suppose that $f(x)$ does not tend to zero as $x \rightarrow \infty$. Then, for some fixed $\epsilon>0$ and all large $\gamma$, the set of $x$-points $\{x \mid x>\gamma, f(x)>\epsilon\}$ has always strictly positive measure. Since $f(x)$ belongs to $L_{1}$ we must conclude that the graph of the "tail" of $f(x)$ which trails towards $+\infty$ must contain an infinite sequence of "spikes" which grow thinner and thinner as we go towards $+\infty$, but which are always of a height greater than $\epsilon$. The density function $h(x)$ will also exhibit these occasional thin "spikes" as $x \rightarrow \infty$.

If $f(x)$ does not satisfy (iii) (we skip (ii) for the moment) then we see, from the proof of the necessity of (iii), that there is no large $\gamma$ such that $h(x)<\gamma$ for all $x>\gamma$. For, if there were such a $\gamma$ then a very slight variant of that necessity argument would show that (iii) is satisfied. Thus, when (iii) is unsatisfied the renewal density is unbounded for all large positive $x$. An example due to Gnedenko and Kolmogorov [5, p. 223] and used by them in another connection provides us with an example of a density which fails to satisfy (iii). They show that if we define a frequency function

$$
\begin{aligned}
g(x) & =\frac{1}{2|x| \log ^{2}|x|} \quad \text { for }|x|<\frac{1}{e} \\
& =0 \quad \text { otherwise, }
\end{aligned}
$$

then there is, for every $n \geqq 1$, a constant $c_{n}>0$ such that 


$$
g^{* n}(x)>\frac{c_{n}}{|x \log (n+1)| x||}
$$

for all $x$ in a neighborhood of the origin. Thus $g^{\dagger}(\theta)$ can be in no class $L_{p}$, for otherwise $g^{*_{n}}(x)$ would be bounded for all $n>p$, which is not the case. Thus we can take $f(x)=g(x-1)$, say, for our example of a density which does not satisfy (iii).

Let us now assume, temporarily, that Theorem 3 has been proved, and turn our attention to constructing a probability density function which does not satisfy condition (ii) of Theorem 1 . For any integer $n$ we can choose $\eta$ such that

$$
1-\frac{1}{2 n^{2}-1}<\eta<1-\frac{1}{2 n^{2}}
$$

Then the function

$$
\begin{aligned}
g(x) & =x-7 & & \text { for } 0<x<1 \\
& =0 & & \text { otherwise, }
\end{aligned}
$$

is in $\beta$ and has a Fourier transform $g^{\dagger}(\theta)$. Furthermore it follows from Titchmarsh [11, p. 172] that there is a constant $\gamma$ such that $\left|g^{\dagger}(\theta)\right|$ $\sim \gamma|\theta| \eta^{-1}$ as $|\theta| \rightarrow \infty$. Thus it appears that $g^{\dagger}(\theta)$ is in $L_{2 n^{2}}$, but in no class $L_{r}$ when $r$ is an integer $\leqq 2 n^{2}-1$.

For $n=1,2, \cdots$, let $f_{n}(x)$ be a function of $\beta$ whose integral is $2^{-n}$, which vanishes outside the interval $I_{n} \equiv\left(n^{-1}-n^{-2}, n^{-1}+n^{-2}\right)$, and whose Fourier transform is in $L_{2 n^{2}}$ but in no class $L_{r}$ when $r$ is an integer $\leqq 2 n^{2}-1$. Such a function $f_{n}(x)$ can obviously be constructed by a suitable shift and scale changes on the example $g(x)$ just discussed.

Define

$$
f(x)=\sum_{n=1}^{\infty} f_{n}(x)
$$

Then $f(x)$ is evidently a probability density function, and it is not difficult to see that $f(x)$ satisfies conditions (i) and (iii) of Theorem 1 (the latter condition for every $\delta>0)$. However, suppose we consider any small interval $(\alpha, \beta)$, $0 \leqq \alpha<\beta$. We can choose large integers $r$ and $n$ such that

$$
\begin{gathered}
\frac{r}{n^{2}}<1, \quad \frac{r}{n^{2}}<\frac{1}{4}(\beta-\alpha), \\
\alpha+\frac{1}{4}(\beta-\alpha)<\frac{r}{n}<\beta-\frac{1}{4}(\beta-\alpha) .
\end{gathered}
$$

Then the function $f_{n}^{* r}(x)$ vanishes outside the interval 


$$
\left(\frac{r}{n}-\frac{r}{n^{2}}, \frac{r}{n}+\frac{r}{n^{2}}\right)
$$

which lies entirely within $(\alpha, \beta)$. If $f_{n}^{* r}(x)$ were bounded then its transform would be in $L_{2}$ and so $f_{n}^{\dagger}$ would be in $L_{2 r}$. But $r<n^{2}$ and so we may conclude that $f_{n}^{* r}(x)$ is not bounded in $(\alpha, \beta)$. But it is clear that $h(x) \equiv \Delta[f(x)] \geqq f_{n}^{* r}(x)$. Thus the renewal density $h(x)$ is not bounded in any interval. Since $f(x)$ satisfies conditions (i) and (iii) of Theorem 1 it appears that condition (ii) must fail to be satisfied.

7. Proof of Theorem 3. For any $n>1$

$$
b_{\delta}^{* n}(x)=\int \cdots \int b_{\delta}\left(z_{1}\right) b_{\delta}\left(z_{2}-z_{1}\right) \cdots b_{\delta}\left(x-z_{n-1}\right) d z_{1} d z_{2} \cdots d z_{n-1} .
$$

If we suppose $x \geqq \alpha+\delta$ then, in order that the integrand in (7.1) be nonzero, there must be an integer $m$ such that (if we define $z_{0}=0$ ), $z_{m-1}<\alpha$ and $\alpha \leqq z_{m}<\alpha+\delta$. Therefore, for $x \geqq \alpha+\delta$,

$$
\begin{aligned}
b_{\delta}^{* n}(x) & =\sum_{m=1}^{n-1} \int_{\substack{\alpha \leq z_{m}<\alpha+\delta \\
z_{m-1}<\alpha}} \ldots b_{\delta}\left(z_{1}\right) \cdots b_{\delta}\left(x-z_{n-1}\right) d z_{1} \cdots d z_{n-1} \\
& \leqq \sum_{m=1}^{n-1} \int_{\alpha \leq z_{m}<\alpha+\delta}^{\ldots} \int b_{\delta}\left(z_{1}\right) \cdots b_{\delta}\left(x-z_{n-1}\right) d z_{1} \cdots d z_{n-1} \\
& =\sum_{m=1}^{n-1} \int_{\alpha}^{\alpha+\delta} b_{\delta}^{* m}(z) b_{\delta}^{*(n-m)}(x-z) d z .
\end{aligned}
$$

For this proof let us write $\Delta\left[b_{\delta}(x)\right]$ more simply as $h_{\delta}(x)$. Then we can conclude from (7.2) that for $x \geqq \alpha+\delta$

$$
\begin{aligned}
h_{\delta}(x) & =\sum_{n=2}^{\infty} b_{\delta}^{* n}(x) \\
& \leqq \sum_{n=2}^{\infty} \sum_{m=1}^{n-1} \int_{\alpha}^{\alpha+\delta} b_{\delta}^{* m}(z) b_{\delta}^{*(n-m)}(x-z) d z \\
& \leqq \int_{\alpha}^{\alpha+\delta} h_{\delta}(z) h_{\delta}(x-z) d z .
\end{aligned}
$$

Now suppose we are given that $h(x)<\gamma$ in $(\alpha, \beta)$. Let us choose $\delta<(\beta-\alpha)$. Then (7.3) proves that

$$
h_{\delta}(x) \leqq \gamma \int_{\alpha}^{\alpha+\delta} h_{\delta}(x-z) d z
$$

for all $x \geqq \alpha+\delta$. But it is well known in renewal theory (and a fact already utilized in the proof of Lemma 7) that 


$$
\int_{x}^{x+\delta} h(z) d z
$$

is a bounded function of $x$. Thus, since $h_{\delta}(x) \leqq h(x)$, it follows from (7.4) that $h_{\delta}(x)$ is bounded for all $x>\alpha+\delta$.

However, $h_{\delta}(x)$ satisfies the integral equation $h_{\delta}(x)=b_{\delta}(x)+b_{\delta} * h_{\delta}(x)$, and so, for $x>\delta$ we have

$$
h_{\delta}(x)=\int_{0}^{\delta} h_{\delta}(x-z) b_{\delta}(z) d z .
$$

Thus, if we assume $y \gg \alpha+\delta$, so that $h_{\delta}(x)$ is bounded in the domain of our discussion,

$$
\sup _{\nu+\delta \leqq x \leqq \nu+2 \delta} h_{\delta}(x) \leqq\left\|b_{\delta}\right\| \sup _{\nu \leqq x \leqq \nu+2 \delta} h_{\delta}(x) .
$$

If we choose $\delta>0$ small enough to make $\left\|b_{\delta}\right\|<1$ then it is clear that the last inequality implies that

$$
\sup _{\nu+\delta \leqq x \leqq \nu+2 \delta} h_{\delta}(x) \leqq\left\|b_{\delta}\right\| \sup _{\nu \leqq x \leqq y+\delta} h_{\delta}(x),
$$

and hence that $h_{\delta}(x) \rightarrow 0$ as $x \rightarrow+\infty$, at an exponential rate. This proves the theorem.

We can thus conclude that if there is no $\delta>0$ such that $\Delta\left[b_{\delta}(x)\right] \rightarrow 0$ then there is no interval to the right of the origin within which $h(x)$ is bounded.

We have incidentally proved:

Corollary 3.1. If $\Delta\left[b_{\delta}(x)\right] \rightarrow 0$ as $x \rightarrow \infty$ then there is some $\eta>0$ such that, as $x \rightarrow \infty$,

$$
\Delta\left[b_{\delta}(x)\right]=O\left(e^{-\eta x}\right) .
$$

As a matter of fact one can show that $\eta=\delta^{-1} \log \left(1 /\left\|b_{\delta}\right\|\right)$; we leave this point as an exercise for the interested reader.

8. Proof of Theorem 4. (i) Let us write

$$
\bar{f}(x)=\phi(x) f(x) /\|\phi(x) f(x)\|,
$$

and $\bar{a}, b_{\delta}, \bar{c}_{\delta}$ for the mutilations of $\bar{f}$ corresponding to the mutilations $a, b_{\delta}, c_{8}$ of $f$. Obviously $\bar{f}(x) \rightarrow 0$ as $x \rightarrow \infty$ if $f(x) \rightarrow 0$. Further, if we write

$$
\lambda=\sup \frac{\phi(x)}{\|\phi(x) f(x)\|},
$$

it is clear that $\left|\bar{c}_{\delta}^{\dagger}\right|<\lambda\left|c_{\delta}^{\dagger}\right|$ and therefore $\bar{c}_{\delta}^{\dagger}$ belongs to any class $L_{p}$ of which $c_{\delta}^{\dagger}$ is a member. Finally we notice that $\Delta\left[b_{\delta}(x)\right] \leqq \Delta\left[\lambda b_{\delta}(x)\right]$ and so, by Lemma $3, \Delta\left[b_{\delta}(x)\right] \rightarrow 0$ as $x \rightarrow \infty$, for all small $\delta$, provided $\Delta\left[b_{\delta}(x)\right] \rightarrow 0$ for some $\delta$. This proves part (i) of Theorem 4. 
(ii) Here we write $\bar{f}(x)=p f_{1}(x)+q f_{2}(x)$ and employ similar notation to that just adopted in (i). Plainly $\bar{f}(x) \rightarrow 0$ as $x \rightarrow \infty$ if both $f_{1}(x) \rightarrow 0$ and $f_{2}(x) \rightarrow 0$. If $c_{1 \delta}^{\dagger} \in L_{p}$ and $c_{2 \delta}^{\dagger} \in L_{q}$ then it is easy to show $\bar{c}_{\delta}^{\dagger} \in L_{r}$, where $r=\operatorname{Max}(p, q, 2)$. Finally we note that

$$
\begin{aligned}
\Delta\left[\bar{b}_{\delta}\right] & =\sum_{n=1}^{\infty} \sum_{r+s=n}\left(\begin{array}{c}
n \\
r
\end{array}\right) p^{r} q^{s} b_{1 \delta}^{* r} * b_{2 \delta}^{* s} \\
& \leqq \sum_{n=1} \sum_{r+s=n}^{\infty} b_{1 \delta}^{* r} * b_{2 \delta}^{* s} \\
& =\Delta\left[b_{1 \delta}\right] * \Delta\left[b_{2 \delta}\right] .
\end{aligned}
$$

But, if $\Delta\left[b_{1 \delta}\right]$ and $\Delta\left[b_{2 \delta}\right]$ belong to $\mathscr{N}$ then, by Lemma $2, \Delta\left[b_{1 \delta}\right] * \Delta\left[b_{2 \delta}\right]$ belongs to $\Re$, and hence $\Delta\left[\bar{b}_{\delta}(x)\right] \rightarrow 0$ as $x \rightarrow \infty$. This proves (ii).

(iii) For this part we write $\bar{f}=f_{1} * f_{2}$. If both $f_{1}$ and $f_{2}$ belong to $\Re$ then, by Lemma $2, \bar{f}$ belongs to $\Re$. If $c_{1 \delta}^{\dagger} \in L_{p}$ and $c_{2 \delta}^{\dagger} \in L_{q}$ then they both belong to $L_{r}$, say, where $r=\operatorname{Max}(p, q, 2)$. Thus $c_{1 \delta}^{\dagger} f_{2}^{\dagger}+c_{2 \delta}^{\dagger} f_{1}^{\dagger}$ also belongs to $L_{r}$ and there is therefore an integer $m$, say, such that $\left(c_{1 \delta} * f_{2}+c_{2 \delta} * f_{1}\right)^{* m}$ is a bounded function. But it is clear that $\bar{c}_{\delta^{*}} \leqq c_{1 \delta} * f_{2}+c_{2 \delta} * f_{1}$, if $\delta^{*}=2 \delta$, so we infer that $\bar{c}_{\delta^{*}}^{* m}$ is also a bounded function. Thus $\bar{c}_{\delta^{*}}^{* m}$ belongs to $L_{2}$, which implies that $\bar{c}_{\delta^{*}}^{\dagger}$ belongs to $\mathrm{L}_{2 m}$. Finally we must show that, for some $\delta>0, \Delta\left[b_{\delta}(x)\right] \rightarrow 0$ as $x \rightarrow \infty$. Denote $\left(f_{1}(x) / 2+f_{2}(x) / 2\right)^{* 2}$ by $\hat{f}(x)$, and denote mutilations of $\hat{f}(x)$ in an obvious way. Then it is clear that $f_{1}(x) / 2+f_{2}(x) / 2$ belongs to $\mathcal{F}$, by part (i) of this proof. Thus $\Delta\left[f_{1}(x) / 2+f_{2}(x) / 2\right]$ is bounded for all large $x$. But it is not difficult to see that $\Delta[\hat{f}(x)] \leqq \Delta\left[f_{1}(x) / 2+f_{2}(x) / 2\right]$ for all $x$. Thus $\Delta[\hat{f}(x)]$ is bounded for all large $x$ and so, by Theorem $3, \Delta\left[\hat{b}_{\delta}(x)\right] \rightarrow 0$ as $x \rightarrow \infty$. However, $\hat{f}=f_{1}^{* 2} / 4+f_{1} * f_{2} / 2+f_{2}^{* 2} / 4$, so that $b_{\delta}(x) / 2 \leqq \hat{b}_{\delta}(x)$, and we have $\Delta\left[b_{\delta}(x) / 2\right] \rightarrow 0$ as $x \rightarrow \infty$. We can now appeal to Lemma 3 to infer that $\Delta\left[b_{\delta}(x)\right] \rightarrow 0$ as $x \rightarrow \infty$ for all sufficiently small $\delta$. This proves the theorem.

9. Concluding observations. Smith [7] discussed also the behavior of $h(x)$ as $x \rightarrow-\infty$. This is equivalent to supposing $\mu_{1}<0$, instead of $\mu_{1}>0$, and still letting $x \rightarrow+\infty$. The only place in our arguments where we have used that $\mu_{1}>0$ is in the proof of Theorem 1 , where we wanted to conclude that if

$$
g(x)=\int_{x-1 / 2}^{x+1 / 2} h(z) d z
$$

then $g(x) \rightarrow \mu_{1}^{-1}$ as $x \rightarrow+\infty$. It is an easy consequence of renewal theory that if we were to have $\mu_{1}<0$ then we would have $g(x) \rightarrow 0$ as $x \rightarrow+\infty$. The rest of the proof of Theorem 1 would hold without change and allow us to conclude that if $\mu_{1}<0$ then conditions (i), (ii), and (iii) of that theorem are necessary and sufficient to have $h(x) \rightarrow 0$ as $x \rightarrow+\infty$.

Feller and Orey [4] have recently shown that $g(x)$ is uniformly bounded and $g(x) \rightarrow 0$ as $x \rightarrow+\infty$ for certain situations in which $\varepsilon X$ cannot be defined. 
It should be clear that we can use our results here also, to conclude that for densities $f(x)$ associated with distributions of the kind envisaged by Feller and Orey, the conditions we give are still necessary and sufficient for the convergence of the renewal density.

In conclusion we make the following observation. If $F(x)$ is the distribution function associated with the probability density $f(x)$ then we have been assuming, of course, that $F(x)$ is absolutely continuous. However, it is possible to prove our theorems assuming that $F(x)$ is absolutely continuous only over the open interval $(0, \infty)$. We can allow $F(x)$ to be singular over $(-\infty, 0]$. Admittedly the renewal density function is no longer defined for negative $x$, but this need not trouble us. We are only concerned with $h(x)$ for positive $x$, and here $h(x)$ remains defined. Perusal of our arguments will show that the only modification needed is to change the $L_{1}$-function $a(x)$ into a certain monotone nondecreasing function $A(x)$, say, and to change all convolutions with $a(x)$ into Stieltjes convolutions with $A(x)$.

\section{REFERENCES}

1. D. Blackwell, Extension of a renewal theorem, Pacific J. Math. 3 (1953), 315-320.

2. S. Bochner and K. Chandrasekharan, Fourier transforms, Annals of Mathematics Studies, No. 19, Princeton Univ. Press, Princeton, N. J., 1949.

3. W. Feller, On the integral equation of renewal theory, Ann. Math. Statist. 12 (1941), 243-267.

4. W. Feller and S. Orey, A renewal theorem, J. Math. Mech. 10 (1961), 619-624.

5. B. V. Gnedenko and A. N. Kolmogorov, Limit distributions for sums of independent random variables, Addison-Wesley, Cambridge, Mass., 1954.

6. W. L. Smith, Asymptotic renewal theorems, Proc. Roy. Soc. Edinburgh Sect. A, 64 (1954), 9-48.

7. - Extensions of a renewal theorem, Proc. Cambridge Philos. Soc. 51 (1955), 629638.

8. - Contributions to Probability and Statistics, pp. 396-413, Stanford Univ. Press, Stanford, Calif., 1960.

9. - On some general renewal theorems for nonidentically distributed variables, to appear in Proc. 4th Berkeley Sympos., Vol. II, pp. 467-514, Univ. of California Press, Berkeley, Calif., 1961.

10. S. Täcklind, Fourieranalytische behandlung vom erneuerungsproblem, Skand. Aktuar. Tidskr. 28 (1945), pp. 68-105.

11. E. C. Titchmarsh, Theory of fourier integrals, 2nd. ed., Oxford University Press, Oxford, 1948.

University of North Carolina,

Chapel Hill, North Carolina 